\title{
Les durkheimiens et le Collège de France
} (1897-1918)

The Durkheimians and the Collège de France (1897-1918)

\section{Rafael Faraco Benthien}

\section{(2) OpenEdition}

\section{Journals}

Édition électronique

URL : http://journals.openedition.org/ress/3345

DOI : $10.4000 /$ ress.3345

ISBN : $1663-4446$

ISSN : 1663-4446

Éditeur

Librairie Droz

Édition imprimée

Date de publication : 15 novembre 2015

Pagination : 191-218

ISSN : 0048-8046

Référence électronique

Rafael Faraco Benthien, « Les durkheimiens et le Collège de France (1897-1918) », Revue européenne des sciences sociales [En ligne], 53-2 | 2015, mis en ligne le 15 novembre 2018, consulté le 20 avril 2019. URL : http://journals.openedition.org/ress/3345; DOI : 10.4000/ress.3345 


\title{
LES DURKHEIMIENS \\ ET LE COLLÈGE DE FRANCE (1897-1918)
}

RAFAEL FARACO BENTHIEN

Universidade Federal do Paraná (Brésil)

rfbenthien@hotmail.com

Résumé. Quoique les premiers durkheimiens n'aient guère eu de difficultés à obtenir des chaires à Paris, le Collège de France leur est resté relativement fermé: sur sept tentatives entre 1897 et 1918, seules deux ont été fructueuses. Pour éclairer cette résistance, d'autant plus difficile à comprendre que le Collège s'ouvrait alors aux sciences sociales, cet article procède en deux temps. Une première partie sur la contextualisation du Collège (sa position au sein du système universitaire, les particularités de son recrutement, les rapports de force le traversant) permet de repérer une série de facteurs dont la régularité est confirmée par l'analyse des candidatures des durkheimiens à laquelle est consacrée une seconde partie.

Mots-clés: Collège de France, durkheimiens, élections, sociologie.

\begin{abstract}
Despite the academic success of the first Durkheimians in Paris, the Collège de France remained refractory to this group: only two out of seven attempts made between 1897 and 1918 were successful. To explain this resistance, difficult to understand since the Collège was becoming more open to the social sciences as a whole during this period, this article proceeds in two stages. A first part provides a contextualization of the Collège (its position within the educational system, the peculiarities of its recruitment, the power relations characterizing the institution) allowing the second part to identify a series of factors whose regularity is confirmed by the analysis of each candidature of the Durkheimians.
\end{abstract}

Keywords: Collège de France, Durkheimians, elections, sociology. 
Depuis les recherches de Victor Karady (1976 et 1979) sur l'institutionnalisation de la sociologie durkheimienne, d'autres travaux, dont les biographies consacrées à Émile Durkheim (Lukes, 1973 et Fournier, 2007) et à Marcel Mauss (Fournier, 1994), ont permis d'éclairer plus précisément la pénétration de cette nouvelle discipline à la Sorbonne ainsi qu'à l'École pratique des hautes études (EPHE)'. Dans ce contexte universitaire, la question des rapports entre les durkheimiens et le Collège de France (ci-après CF) a été beaucoup moins étudiée, alors même qu'elle s'avère pourtant problématique ${ }^{2}$. En effet, au moment où le Collège s'ouvre aux sciences sociales (tableau i), Durkheim et ses collaborateurs ne ménageront pas leurs efforts pour s'y faire une place : six candidatures en témoignent (tableau 2). Or parmi elles, les deux seules qui ont débouché sur un recrutement relevaient de disciplines traditionnelles rattachées aux «études littéraires», et non des sciences sociales.

Tableau I. Les sciences sociales au CF (1897-1918)

\begin{tabular}{|l|c|}
\hline \multicolumn{1}{|c|}{ CHAIRE } & CRÉATION \\
\hline Philosophie sociale & 1897 \\
\hline Sociologie et Sociographie musulmane & 1902 \\
\hline Histoire du travail & 1907 \\
\hline Étude des faits économiques et sociaux & 1912 \\
\hline Géographie humaine & 1912 \\
\hline Prévoyance et Assistance sociale & 1917 \\
\hline
\end{tabular}

Tableau 2. Candidatures au CF jusqu’à I9ı 8

\begin{tabular}{|l|c|c|}
\hline \multicolumn{1}{|c|}{ CANDIDAT } & ANNÉE & CHAIRE \\
\hline Durkheim & 1897 & Philosophie sociale \\
\hline Meillet & 1906 & Grammaire comparée \\
\hline Fossey & 1906 & Philologie et Archéologie assyriennes \\
\hline
\end{tabular}

I Voir à ce sujet Mucchielli, 1998; Strenski, 2000 et Brooks, 2002.

2 L'unique texte répertorié sur cette question est une note publiée par Besnard (1979). Les candidatures de Mauss et de Durkheim ont aussi, bien entendu, fait l'objet d'investigations par leurs biographes respectifs. 


\begin{tabular}{|l|c|c|}
\hline Mauss & 1907 & Histoire des religions \\
\hline Mauss & 1909 & Histoire des religions \\
\hline Simiand & 1912 & Étude des faits économiques et sociaux \\
\hline
\end{tabular}

Pour mieux comprendre cet échec, une première partie consacrée à la contextualisation du CF (sa position au sein du système universitaire, les particularités de son recrutement, les rapports de force le traversant) permet de repérer une série de facteurs de résistance en son sein dont la régularité est confirmée, dans une seconde partie, par l'analyse des candidatures de Durkheim et de ses collaborateurs de L’Année Sociologique. Cette structure du texte a pour objectif de souligner tant le poids des variables à proprement parler «scientifiques» (la hiérarchie des disciplines et objets) que l'importance des enjeux non scientifiques (confessionnels et surtout politiques) dans l'histoire de l'institutionnalisation de cette nouvelle discipline.

\section{LE COLLÈGE DE FRANCE : HISTOIRE ET «DISPOSITIONS» D'UNE INSTITUTION}

\section{I.I LE COLLÈGE DE FRANCE ET L'INNOVATION UNIVERSITAIRE}

L'étude d'une institution comme le CF ne peut faire l'économie de celle de sa genèse. La création, en I530, du Collège royal résultait des mouvements littéraires et scientifiques à la cour de François I ${ }^{\text {er }}$, très critiques face aux institutions de l'enseignement médiéval3. Se détournant de la scolastique et en affinité avec l'air du temps des réformes religieuses, le Collège se vouait alors aux sciences et à l'étude des langues. Aux premières chaires de grec et d'hébreu, viendront s'ajouter au cours du temps et jusquau XVII siècle de nouvelles disciplines : l’arabe, le syrien, l'éloquence latine, la philosophie antique, les mathématiques, la médecine et le droit. L'innovation portait tant sur le contenu que sur la forme. Contrairement aux universités, le CF ne possédait pas de programmes fixes et ne délivrait pas de diplômes. Il s'agit donc de la première institution française qui a pu impulser d'importantes innovations et rompre avec le monopole des corporations catholiques ${ }^{4}$.

3 Voir notamment Durkheim, 1999 (1904-1905), p. 134-260. Voir aussi Lefranc, 1893.

4 On peut voir là l'une des constantes des réformes du système éducatif en France, tendant à se traduire par des innovations à la périphérie du système avant que le déséquilibre ainsi 
Cette autonomie accordée par le pouvoir central ne s'est jamais démentie. Sans doute, l'élan réformateur des premières années s'est plusieurs fois ralenti, soit que les représentants de l'État se soient montrés plus sensibles aux demandes des scientifiques et des littéraires plus orthodoxes, soit du fait d'une moindre cohésion entre les mouvements «d'avant-garde». Sans pour autant devenir une institution conventionnelle, le CF a dû céder à l'intégration de représentants de l'establishment. La période antérieure à la Révolution lui a cependant valu un prestige accru tant le CF sut s'imposer comme une alternative au modèle universitaire alors en vigueur. Pendant la Révolution, la première École normale et les Écoles centrales elles-mêmes cherchèrent à y puiser des principes pour rompre avec des parcours surchargés par le latin et la rhétorique.

Si les réformes profondes du système d'enseignement resteront inabouties, le CF renforcera sa position au cours de la première moitié du XIX siècle dans la mesure où il demeure une référence en matière d'audace et d'originalité. Mais c'est surtout un peu plus tard, entre I860 et I880, qu'il atteint son apogée. Selon Charle (1997), sous la pression de la référence à l'Allemagne, le CF devient le siège des «maîtres à penser» de disciplines nouvelles. Marcelin Berthelot en chimie, Théodule Ribot en psychologie, Michel Bréal en linguistique : à la pointe de ce qui se faisait de plus moderne dans leur domaine, tous possédaient déjà une renommée internationale. Certes, les penseurs «mondains» et plus orthodoxes n'étaient pas en reste. Pourtant, c'est au premier groupe que le gouvernement de la Troisième République a recours pour moderniser l'enseignement supérieur. Aussi, l'élection au CF symbolise à cette époque le couronnement d'une carrière réussie.

Dans les décennies suivantes, la place dominante du CF en tant que vivier de l'innovation n'est cependant plus aussi assurée en raison des réformes qui seront effectuées à la Sorbonne, mais également du fait de la création de l'EPHE. Fondée en I868 en tant qu'institut de recherches, cette institution se structure autour de séminaires, créant les conditions pour la professionnalisation de

créé ne conduise à réajuster l'ensemble. Pour l'Ancien Régime, voir Grell, 1995, p.45-143. Pour le XIXe siècle, voir Ringer, 1992, p.40-93. 
plusieurs domaines de la connaissance. Étant donné l'intérêt constant du CF pour les disciplines enseignées à l'EPHE, on comprend qu'il ait été difficile à cet institut de conserver ses enseignants. Il n'était pas rare que plusieurs d'entre eux se portent candidats pour un poste analogue au CF, attirés par de meilleurs salaires, une charge d'enseignement moins lourde et un plus grand prestige. Ce rôle de véritable vivier à professeurs de l'EPHE n'en a pas moins fait une institution rivale, capable de former des concurrents de taille face aux titulaires du CF.

La réforme de la Sorbonne a représenté une menace plus importante. Cette institution ancienne a alimenté jusque dans les années i880 l'idéal de l'«honnête homme», de formation générale à caractère littéraire. Elle répondait ainsi aux demandes des lycées et des facultés, qui en partageaient les valeurs. Pour briser ce cercle, les partisans de la science décidèrent d'introduire à la Sorbonne des chercheurs réputés susceptibles de la transformer en centre de recherches proposant une autre formation, dans le cadre laïque et républicain. Les réformes de 1902 mettent fin au monopole du latin comme condition d'accès à l'enseignement supérieur en introduisant les langues modernes et les sciences comme matières pouvant le remplacer au baccalauréat. Ces changements atténuent aussi la séparation entre universités et Grandes Écoles, les élèves de l'ENS pouvant par exemple suivre les cours de la Sorbonne. Toute la gamme des savoirs proposés est affectée. Les nouveaux idéaux de l'institution sont alors incarnés par des personnalités comme Émile Durkheim, Charles Seignobos, Gustave Lanson - autant d'esprits originaux et novateurs dans leur domaine. La Sorbonne commence ainsi à entretenir un niveau d'excellence en matière d'innovation (Ringer, 1992, p. 196-237).

Tandis que le CF était bien l'unique lieu dans lequel l'hétérodoxie pouvait triompher jusqu’aux années i860, la situation s’est modifiée avec la création de l'EPHE. Si, jusque dans les années I880, coexistent une Sorbonne «mondaine » et une EPHE «scientifique », la réorganisation de l'université altère cet équilibre. Les réformes dans l'enseignement et la question du rôle respectif des nouveaux et des anciens savoirs ont mobilisé, dans un sens comme dans l'autre, d’amples secteurs de la société, mettant le CF au cœur des débats. Ceci explique pourquoi les candidatures des sociologues ont pu susciter des combats, ne mobilisant pas seulement des arguments intellectuels. 


\section{I.2. LE MODE DE RECRUTEMENT DU COLLÈGE DE FRANCE}

En ce qui concerne les spécificités du recrutement au CF, deux règles ont été observées dès l'origine : d'une part, quand une chaire se retrouvait vacante à la suite d'un décès ou d'un départ à la retraite, les professeurs devaient débattre pour savoir si cette chaire devait être maintenue ou transformée; d'autre part, aucune condition de diplômes n'était imposée aux candidats. Au tout début, cette ouverture s'expliquait par l'impératif de recruter des spécialistes de savoirs encore dépourvus de formation universitaire. Cet usage persistant permit cependant aussi à des personnalités originales de tenter la consécration.

Sauf exception, toute décision relative au recrutement dépend du vote de l'assemblée des enseignants. Le vote a lieu à bulletin secret après une présentation des candidats suivie de délibérations. À partir de la fin du XIX siècle au moins, l'assemblée devait proposer deux noms au ministre de l'Instruction publique.

À l'issue d'une procédure pouvant demander plusieurs tours, en fonction des divisions des professeurs, le nom d'un premier candidat est désigné à la majorité des voix. On disait de ce candidat, jugé par l'assemblée comme étant le plus apte, qu'il était placé en «première ligne». Cette élection faite, un nouveau vote vise alors à désigner un autre nom parmi les candidats, inscrit cette fois-ci en «deuxième ligne».

De manière générale, le ministère suivait le premier choix de l’assemblée. Mais il restait possible de nommer le candidat présenté en «deuxième ligne». Une volonté politique suffisamment affirmée permettait de le faire. En outre, à titre exceptionnel, l'État pouvait nommer un titulaire sans vote préalable. Il s'agissait toutefois d'une prérogative particulière, limitée à la désignation du premier titulaire d'une chaire nouvellement créée, dont les dirigeants politiques ne faisaient pas nécessairement usage ${ }^{5}$. Enfin, une troisième voie d'entrée était possible: si un mécène choisissait de financer une chaire pour un chercheur spécifique, il suffisait alors de l'aval de l’assemblée. Le titulaire de la nouvelle chaire ne possédait cependant pas de droit de vote. 
Quant aux candidats, deux options se présentaient à eux: ou bien ils avaient des parrains politiques capables de les faire nommer sur une nouvelle chaire, ou bien ils devaient entrer en campagne pour prendre possession d'une chaire déjà existante. Comme l'État ne créa que cinq chaires entre I897 et 1918, cette seconde situation fut la plus fréquente. Dès l'annonce d'un poste vacant ou créé, le candidat devait adresser une lettre expliquant ses motivations, accompagnée de son curriculum vitae, à l'administrateur du CF. Ensuite, rendre visite aux membres du CF était de mise. De l'annonce de la candidature à l'élection, chacun devait activer son réseau de relations car il était difficile à un candidat de concourir pour la «première ligne» sans aucun appui interne préalable. On pouvait aussi s'informer sur les positions des votants auprès des cercles de chacun. Tous s'efforçaient de faire des pronostics, recensant des promesses de vote ou des déclarations d'hostilité. Ces procédures n'excluaient pas non plus les campagnes diffamatoires. Il s'agissait donc de batailles où toute forme de naïveté ou d’ambition démesurée était payée au prix fort.

\section{I.3. LES RAPPORTS DE FORCE AU COLLÈGE DE FRANCE}

Qui évaluait les sociologues? Quelles tensions ou affinités sont apparues à l'occasion de ces élections? Répondre à ces questions exigerait de considérer la totalité des variables impliquées dans chaque cas. Renforcée par le caractère secret du vote, la diversité des paramètres concernés empêche de concevoir un modèle omni-explicatif. Cependant, un certain nombre de régularités se dégagent dès lors que l'on tient compte des catégories dans lesquelles les rapports de force étaient décrits à l'époque.

Un premier principe de classement distingue les «scientifiques» des «littéraires ». Typiquement française, cette distinction découle des réformes mises en place pendant et après la Révolution. L'université avait conservé jusqu'alors la structure héritée du XIII ${ }^{\mathrm{e}}$ siècle, à savoir les Facultés de droit, de médecine, de théologie et des arts. À partir de I808, l’avancée des sciences et le poids significatif de la littérature française conduisent à subdiviser les Facultés des arts en deux institutions juridiquement équivalentes aux autres : les Facultés de sciences et les Facultés de lettres. Resté en marge du système 
universitaire de l’Ancien Régime, le CF est néanmoins touché par cette scission et cherche à donner de l'espace à ce qu'il y avait de plus novateur dans les nouvelles facultés. À la fin du XIX ${ }^{\mathrm{e}}$ siècle, ces catégories apparaissaient déjà si clairement établies que Renan notait la tendance des «scientifiques » du CF à «mieux » voter quand l'élection concernait les «littéraires », et réciproquement (voir Charle, 1997). Mais il ne s’agissait là que de qualité du vote : étant donné les divisions existantes à l'intérieur de chacune de ces communautés, il était courant que le soutien d'un groupe pèse sur l'élection d'une chaire de l'autre groupe. La répartition des chaires du CF entre I897 et I9I8 permet d’ailleurs d'observer un équilibre de forces entre les deux pôles.

Tableau 3. «Scientifiques» et «littéraires» au CF (en nombres absolus et relatifs)

\begin{tabular}{|l|c|c|c|c|}
\hline & 1897 & 1904 & 1911 & 1918 \\
\hline Chaires de sciences & $11(26,8 \%)$ & $12(27,3 \%)$ & $14(30,4 \%)$ & $13(28,3 \%)$ \\
\hline Chaires de lettres & $30(73,2 \%)$ & $32(72,7 \%)$ & $32(69,6 \%)$ & $33(71,7 \%)$ \\
\hline Total & $41(100 \%)$ & $44(100 \%)$ & $46(100 \%)$ & $46(100 \%)$ \\
\hline
\end{tabular}

Une seconde distinction, relative à l'organisation de l'Institut de France, retient l'attention. Créé pendant la Révolution, l'Institut réunit sous le même toit cinq académies: l’Académie française (AF), l’Académie des sciences (AS), l'Académie des beaux-arts (ABA), l'Académie des inscriptions et belles-lettres (AIBL), et l'Académie des sciences morales et politiques (ASMP). Sans aborder l'histoire de ces institutions, avant tout dévolues au mécénat et à la promotion des sciences, il faut noter qu'elles n'ont jamais entretenu de liens directs avec l'enseignement. Mais compte tenu de leur prestige, il n'était pas rare qu'un membre du CF appartînt au moins à l'une de ces académies.

Ces divisions nous intéressent puisque ce sont elles qui marquaient alors la perception institutionnelle des frontières entre les savoirs. L'AS regroupait plutôt les scientifiques. À l'AF et à l'ABA, on retrouvait les artistes et critiques en général. L’AIBL réunissait, pour sa part, les spécialistes en langues et littératures, les archéologues et les historiens. Enfin, l’ASMP accueillait les philosophes et tous ceux qui proposaient de penser les questions politiques et sociales de 
façon théorique, descriptive ou normative. C'est dans ce nouvel espace, qui pouvait paraître suspect aux yeux des responsables politiques et spécialistes des autres disciplines, que la sociologie se devait de trouver sa place ${ }^{6}$. Dans la pratique, entre I897 et I9I8, on constate que les chaires en rapport avec l'AIBL constituent le groupe le plus nombreux au CF, avec i8 (en I897, 43,9\% au total) à 20 (en I9r $8,43,5 \%$ au total) chaires. Les disciplines correspondant à l'ASMP et l'AS représentaient chacune un quart des postes.

Tableau 4. Composition du CF : les académies (en nombres absolus et relatifs)

\begin{tabular}{|l|c|c|c|c|}
\hline & 1897 & 1904 & 1911 & 1918 \\
\hline Chaires en lien avec l'AIBL & $18(43,9 \%)$ & $19(43,2 \%)$ & $20(43,5 \%)$ & $20(43,5 \%)$ \\
\hline Chaires en lien avec l'AS & $11(26,8 \%)$ & $12(27,3 \%)$ & $14(30,4 \%)$ & $13(28,3 \%)$ \\
\hline Chaires en lien avec l'ASMP & $10(24,4 \%)$ & $11(25 \%)$ & $11(23,9 \%)$ & $12(26,1 \%)$ \\
\hline Chaires en lien avec les Arts & $2(4,9 \%)$ & $2(4,5 \%)$ & $1(2,2 \%)$ & $1(2,2 \%)$ \\
\hline Total & $41(100 \%)$ & $44(100 \%)$ & $46(100 \%)$ & $46(100 \%)$ \\
\hline
\end{tabular}

Cette même division se fera sentir sur les élections au CF. Dans un premier temps, cela rendait plus facile la vie des candidats qui n'étaient pas considérés comme des «purs» représentants d'un domaine spécifique. En effet, la simple érudition littéraire risquait de susciter l'hostilité des scientifiques. De la même manière, le scientifique dépourvu de formation générale n’aurait pas manqué d'alimenter l'antipathie des littéraires. Il est intéressant d'observer cependant que ces pôles pouvaient se retrouver à l'intérieur de chacune des divisions des savoirs. Parmi les hommes de lettres, on en retrouvait qui pouvaient se rapprocher des scientifiques, tout comme on voyait chez les scientifiques des individus plus sensibles à l'érudition. Quant aux candidats qui se rattachaient aux disciplines ressortissant à l'ASMP, ils se retrouvèrent eux-mêmes dans une position ambiguë, située entre la science, l'érudition et la politique.

6 Cette nouvelle Académie a été créée pendant la Révolution française à l'initiative des «idéologues», intéressés par la confluence des études scientifiques des mœurs et de la vie politique. Elle innovait donc par rapport à ce qui se faisait à l'intérieur les académies plus anciennes, et notamment à l'AIBL, encore très liée à des programmes littéraires ou purement érudits. Pour une vision d'ensemble concernant la sociologie, voir la première partie de Heilbron, 2006. 
Néanmoins, les catégories de l'entendement professoral ne s'alimentaient pas que de facteurs intellectuels ou institutionnels. Commentant sa campagne en 1944, Maurice Halbwachs brossait le tableau des rapports de force à l'œuvre au sein du CF dans les termes suivants :

D’après ce que j’ai appris de divers côtés [...], voici ceux qui ont dû voter pour moi : Piéron, Tonnelat, Gabriel, Piganiol, - Grenier, Pelliot, Massignon, Maspero, Joliot, - Lacassagne, Roques (tous ceux-là, soit 12, certainement), Fauré-Frémiet, Coornaert, Robert (?), Chassigneux, Faral (?) - soit 17.

Ont voté contre moi pour Gouhier, Gilson, Lavelle, Baruzi, Mazon, Hazard, Siegfried, Lacau (?), Fallot, Defraisse, de Broglie, Duclaux (?), Valéry (?) - soit I2.

Febvre a dû voter seul au deuxième tour pour Renaudet.

Piéron croit que Faral a voté contre moi, pour Gouhier, pour des raisons administratives, - craignant que mon élection ne soulève des difficultés côté ministère. Cela m'étonnerait. Impossible de percer ce mystère.

En somme j’ai eu contre moi les philosophes catholiques, l’Académie française, les cléricaux, l'unique représentant (grand bourgeois) de l'Académie des sciences morales et politiques (dont je suis correspondant), les scientifiques réactionnaires.

J’ai eu pour moi presque tous les linguistes, les savants spécialistes des diverses civilisations (sauf Mazon), les historiens, les scientifiques avancés, surtout quatre biologistes (ou médecins). Il m’a manqué deux absents, Przyluski et Wallon (qui était pour moi, mais dont le cours est suspendu), - et aussi (d’après Piéron) Courrier : soit trois absents. [...]

En somme, philosophie métaphysique et religieuse, littérature pure, sciences des brutiers, - contre histoire sociologique, psychologie scientifique, linguistique, et biologie (1999, p. 227).

Si les divisions de l'Institut ou l'opposition sciences/lettres aident donc à mieux interpréter les élections, on peut voir, en lisant Halbwachs, que leur importance dépend de leur combinaison avec d’autres facteurs. L’origine sociale, l'appartenance religieuse et les réseaux politiques ont aussi pu influencer les électeurs.

Grâce aux données présentées par Charle et Telkès (1988), on sait aujourd'hui, qu’entre I870 et 19I4, la morphologie des membres du CF était plutôt hétérogène. Même si les catholiques restaient majoritaires, nombreux étaient issus de minorités confessionnelles. Les orientations politiques couvraient aussi un 
large éventail. De quelques monarchistes à certains socialistes, le paysage politique se répartissait en grande majorité entre les républicains libéraux et les conservateurs. Cette situation plutôt instable laissait la porte ouverte à une série d'alliances et de conflits. De ce point de vue, le CF se distinguait clairement de la Sorbonne où les minorités religieuses et les professeurs de gauche étaient relativement plus nombreux (Charle, 1985).

\section{LES SIX CANDIDATURES DES DURKHEIMIENS AU CF}

Durkheim et quatre autres collaborateurs de L'Année Sociologique se porteront candidats pour occuper une chaire au CF entre i897 et 1918. Chacune de ces postulations illustre une combinaison particulière des facteurs épistémologiques, institutionnels et sociaux mis en évidence dans la partie précédente. Toutefois, les variables les plus lourdes semblent avoir été de nature confessionnelle et, surtout, politique. Présente dès le début, bien qu'en sourdine, elles ne feront que gagner en ampleur.

\section{I.DURKHEIM: ÉCHECS PARADIGMATIQUES}

La première candidature d'un sociologue au CF fut celle d'Émile Durkheim. Il se présente en I897, avant d'être incité à le faire officieusement une seconde fois, sept ans plus tard. Cette première candidature est motivée par la décision gouvernementale de créer une chaire intitulée «Philosophie Sociale». Peu avant, l'organisation des forces politiques de gauche avait déjà mis au premier plan les questions sociales ${ }^{7}$. Les responsables de cette création inscrivaient leur action dans ce débat. Trois candidats se présentent pour le poste. Durkheim, le plus jeune, avait déjà accumulé des titres non négligeables : normalien, agrégé de philosophie et docteur ès-lettres, il occupait la chaire de «Science sociale et Pédagogie » à la Faculté des lettres de Bordeaux. Parmi ses publications, on peut citer De la division du travail social (1893), Les Règles de la méthode sociologique (I894) et Le Suicide (I897), qui venait tout juste de paraître.

7 Nous pensons, notamment, aux grèves au nord de la France et au début de négociation qui aboutiront, en 1905, à la fusion de plusieurs partis politiques de gauche avec la création de la Section française de l'Internationale ouvrière. 
Les deux autres candidats étaient Alfred Espinas et Jean Izoulet. Le premier, né en I844, était lui aussi normalien et agrégé de philosophie. Son parcours était celui d’un enseignant au lycée puis à l'université. À la Faculté des lettres de Bordeaux, où il exerçait depuis 1878, Espinas avait précédé Durkheim en tant que titulaire de la chaire de «Pédagogie», assumant aussi des postes administratifs, dont celui de recteur. Il n'obtint un poste à la Sorbonne que seize ans plus tard, quand il fut désigné pour la chaire d'«Histoire de l'économie sociale ». À une reconnaissance intellectuelle et institutionnelle considérable s'ajoutait le fait que sa thèse, Les Sociétés animales, soutenue en I877, était considérée comme le texte fondateur de la sociologie scientifique en France. Des trois candidats, il était celui dont la réputation était la plus affirmée. Pour ce qui est des critères intellectuels, Izoulet, en revanche, donnait l'impression de n’avoir aucune chance face à ses adversaires : né en I854, normalien et agrégé de philosophie, il s'était consacré à l'étude de la pensée sociale prérévolutionnaire. Professeur au lycée Condorcet, son œuvre la plus connue était La Cité moderne et la métaphysique de la sociologie (1897 [1894]).

Dans sa correspondance personnelle, Durkheim se montre sceptique sur ses chances (Fournier, 2007, p. 343-346). Du fait de difficultés budgétaires remettant en question la création de cette chaire, les responsables du gouvernement écartent finalement Espinas et Durkheim, représentant l'un et l'autre des courants de pensée et des affiliations trop hétérodoxes aux points de vue religieux et politique ${ }^{8}$. C'est donc le candidat le moins brillant mais le plus proche de la bourgeoisie traditionnelle qui est retenu'. Le choix d'Izoulet, qui se fit connaître par la suite pour ses attaques contre L’Année sociologique, ne donnait pas seulement le la d'une hostilité vouée à se renforcer de la part du CF envers les durkheimiens. Il faisait encore de l'échec de Durkheim un échec paradigmatique.

8 Espinas était un athée convaincu alors que Durkheim venait d'une famille juive. Quant à la dimension politique de la nomination, Besnard (1979) aura montré que les parlementaires souhaitaient la nomination d'une personne clairement opposée au socialisme.

9 L'obéissance d'Izolet à la pensée catholique a été soulignée à l'époque, ainsi que son antimarxisme, voir Sabatier, 1898. Nous remercions le rapporteur choisi par la Revue européenne des sciences sociales d'avoir attiré notre attention sur ce texte. 
Sans donner lieu à une candidature officielle, une seconde occasion se présente à Durkheim avec la mort de Gabriel Tarde, en 1904. Titulaire de la chaire de «Philosophie moderne», interlocuteur et adversaire des durkheimiens, Tarde était en ce début de siècle l'une des références sur les questions sociales ${ }^{10}$. Durkheim était alors l'un des rares chercheurs à pouvoir faire valoir une carrière et une renommée suffisantes pour le remplacer. De fait, au cours de ces dernières années, son prestige académique s'était encore accru: non seulement on lui attribuait le succès de la revue qu'il dirigeait, mais il occupait encore, comme suppléant, la chaire de «Science de l'éducation» à Paris. Syy ajoutait le rôle qu'il avait joué comme membre fondateur de la Ligue des Droits de l'Homme et comme dreyfusard.

La situation connaît plusieurs rebondissements. Tout d'abord, ce n'est pas par un recrutement que la chaire est pourvue: Henri Bergson, qui occupe celle de Philosophie moderne, abandonnait celle de «Philosophie grecque et latine». Or, si plusieurs candidats se présentent pour la chaire qu'il laissait vacante, les professeurs votent, le 28 novembre 1904, en faveur de sa transformation (à 24 voix contre I0). Cinq propositions sont faites: «Histoire générale», «Philosophie générale», «Antiquités nationales», «Pédagogie» et «Sociologie». Cette dernière avait pour avocat Pierre Janet, professeur de «Psychologie expérimentale et comparée » et ancien camarade de Durkheim à l'ENS. Les sources d'archives le confirment : face à l'assemblée, Janet

demande un enseignement nouveau, celui de la sociologie qui a un caractère philosophique et jette aujourd'hui un vif éclat; il y a tel savant qui a appliqué une méthode vraiment scientifique et qui fait école. Il serait intéressant de lui ouvrir les portes du Collège. La Chaire de Sociologie serait dans le groupe des sciences morales et ne modifierait pas l'équilibre du Collège (voir Archives du CF, l'Assemblée du 28 novembre 1904 [G-IV-g2IR]).

Henri Bergson et Alfred Le Chatelier, professeur de «Sociologie et de Sociographie musulmane», adhèrent à la proposition. Contre eux, trois antiquisants (René Cagnat, Gaston Boissier et Louis Havet) et un germaniste (Arthur Chuquet) plaident en faveur de l'«Histoire générale » et des «Antiquités nationales », insistant sur la nécessité d’un travail d'érudition plus spécialisé en 
ce domaine. Le procès-verbal ne livre aucun indice sur la personne qui aurait proposé la chaire de «Pédagogie». On y relève que le seul à suggérer l'option «Philosophie générale » est Michel Bréal. Deux tours de vote ne départagent pas les principales propositions : la «Sociologie», l'«Histoire générale» et les «Antiquités nationales» recueillent onze voix chacune.

Durkheim, que Bergson et Janet interrogent sur ses intentions, connaissait leur engagement à son égard. Mais le contexte lui apparaissait peu favorable, comme en témoignent les lettres à son neveu (1998, p. 354-355). Le I5 janvier 1905, l'assemblée reprend ses débats. À cette occasion, Bergson s'engage en faveur de la sociologie et évoque implicitement Durkheim:

La sociologie, comme [Durkheim] la conçoit, est une dépendance des sciences historiques et des sciences positives. Elle ne prétend appliquer ses recherches quau passé, mais elle a en réalité des applications pratiques, établit entre les faits des relations qu'on ne soupçonne pas. Elle a donnée naissance à une publication importante, L’Année sociologique (voir Archives du CF, Assemblée du I5 janvier 1905 [G-IV-g22M]).

Il ajoute que ce savoir n’avait pas été accepté à la Sorbonne ${ }^{11}$. Grégoire Wyrouboff, professeur d'«Histoire générale des sciences » et positiviste notoire, déclare alors son soutien à cette proposition.

Mais ce discours ne convainc pas les adversaires. Tout indique que les partisans de l'«Histoire générale » et des « Antiquités nationales» se sont mis d’accord avant le vote. Boissier et Cagnat reformulent leurs demandes et proposent une chaire intitulée «Histoire et Antiquités nationales». Les arguments appuyant ce changement étaient de même nature que ceux de Bergson : il s’agissait d’une science nouvelle, positive et sans représentation à la Sorbonne. La proposition reçut un appui important d’Albert Réville, pasteur protestant et professeur d'«Histoire des religions ». Enfin, à contre courant, l'helléniste Paul Foucart propose un nouveau vote pour maintenir la chaire de «Philosophie ancienne». Au terme des débats, les résultats sont nets : sur 33 votants, on décompte I vote nul, i9 votes en faveur

II La chaire de Durkheim à Paris ne fut en effet transformée en «Science de l'éducation et sociologie» qu'en 1913. 
d'«Histoire et Antiquités nationales», 2 pour la «Philosophie grecque et latine» et, comme à la dernière assemblée, i en faveur de la «Sociologie».

Il est difficile d'identifier avec précision les votants pour ou contre la sociologie. Aucun motif politique n’a été évoqué. Les membres de l’ASMP étaient divisés, alors qu'il s'agissait d'une chaire relevant de leur domaine. Bergson et Janet optèrent pour la sociologie. Dans ses lettres à Mauss, Durkheim laisse entendre qu'il aurait eu le soutien de Paul Leroy-Beaulieu et Émile Levasseur (Durkheim, 1998, p. 354-355). Réville et Izoulet prirent une position contraire. Parmi les spécialistes de langues et civilisations, l'unique défenseur reconnu aura été Sylvain Lévi. Chez les scientifiques, seul Wyrouboff se prononça. La position majoritaire reçut ainsi l'appui de tout le gros de l'AIBL, ainsi qu'une grande partie de l'ASMP.

\subsection{MEILLET: UN SUCCÈS «LÉGITIME»}

Un an après le deuxième échec de Durkheim, les candidatures de deux chercheurs issus des rangs de L'Année sociologique furent accueillies: Antoine Meillet et Charles Fossey sont élus le 17 décembre 1905, mais dans des circonstances différentes.

Meillet se présente pour la chaire de «Grammaire comparée», dont le titulaire, Bréal, venait de prendre sa retraite. Âgé de 39 ans à l'époque, Meillet avait fait ses études à la Sorbonne et passé l'agrégation de grammaire (I889, I ${ }^{\mathrm{e}}$ place). Il n'était pas inconnu au CF puisqu'il avait déjà été suppléant de Bréal entre I899 et 1900. Il était surtout titulaire à l'EPHE d'une chaire également intitulée «Grammaire comparée ». Meillet dominait les langues slaves comme très peu, l'iranien ancien et l'arménien, ainsi que les langues grecque et latine. Il s'agissait là d’un successeur légitime.

L’adhésion de Meillet à la sociologie était alors publique. Ses premiers contacts avec cette science venaient de l'EPHE, par l'intermédiaire de Mauss et d'Henri Hubert. À partir de 1902, il commence à collaborer à L’Année sociologique. Envisager le langage comme un fait social était un des points fondamentaux de son programme. Par l'importance qui lui était reconnue dans son domaine, il aura apporté du prestige et quelques contributions au projet porté par Durkheim. 
Lors de sa campagne au $\mathrm{CF}$, un seul autre candidat s'oppose à Meillet: Maurice Grammont, spécialiste de la langue française. Ce dernier, en marque de respect, insiste pour que son nom ne soit considéré que pour la « deuxième ligne». Meillet s'impose alors avec 33 voix sur 34 (on compte un vote nul) ${ }^{12}$. Mais s'il fut élu à l'unanimité, sa leçon inaugurale allait susciter de vives réactions. Devant un amphithéâtre bondé, le I3 février 1906, il adresse des éloges à la sociologie au détriment de la méthode historique :

Mais il importe plus d'indiquer les problèmes nouveaux que pose le progrès de la science que de répéter les solutions, d'ailleurs toujours incomplètes, qu’ont reçues les vieux problèmes [...] Le XIX ${ }^{e}$ siècle a été le siècle de l'histoire, et les progrès qu'a réalisés la linguistique en se plaçant au point de vue historique ont été admirables; les sciences sociales se constituent maintenant, et la linguistique y doit prendre la place que sa nature lui assigne (Meillet, 1948, p. I8).

La réponse ne se fit pas attendre. Une note publiée dans la Revue des études anciennes décrie la naïveté du soutien de Meillet à la sociologie, une science peu consolidée si on la compare au savoir précis que peut représenter l'histoire (Bourciez, 1906). La critique n’affectera en rien sa réputation en tant que linguiste, ses œuvres lui valant une reconnaissance non disputée. De plus, une certaine discrétion en ce qui concernera ses positions politiques le garde à distance du militantisme de beaucoup de durkheimiens. Sa leçon inaugurale n’aura cependant pas facilité l'apaisement de ceux qui, en voulant maintenir la supériorité des savoirs traditionnels, regardaient les sociologues avec méfiance.

\subsection{FOSSEY: UN SUCCÈS «ILLÉGITIME»}

À la suite du décès de Jules Oppert, titulaire de la chaire de «Philologie et d’Archéologie assyriennes », la même assemblée à avoir élu Meillet choisit Charles Fossey pour ce poste. Cette élection ouvre une grande controverse. Âgé alors de 36 ans, Fossey avait un cursus honorable. Ancien élève de l'ENS et de l'EPHE, ancien membre de l'École française d'Athènes (1894-1897) et de l'Institut français d’archéologie orientale du Caire (I897-1899), il oriente d’abord sa carrière vers l'hellénisme avant de se tourner vers l'orientalisme. Son premier 
poste, à l'EPHE, était déjà relié aux anciennes sociétés du Moyen Orient, et plus spécialement aux croyances magico-religieuses. Il s'est aussi fait remarquer pour avoir publié le premier manuel d’assyriologie français (1904).

C'est des premières années de son parcours que datent ses contacts avec les sociologues à l'EPHE. Mais son adhésion au groupe fut plutôt modeste. Fossey n'écrivit que quatre comptes rendus pour les sixième et septième volumes de L'Année sociologique, tous en relation avec les mythes et rites babyloniens. Il participa, par ailleurs, au projet coordonné par Henri Hubert et Isidore Lévy pour traduire le Manuel d'histoire des religions de Chantepie de La Saussaye (1904).

Trois candidats se présentèrent contre Fossey: Joseph Halévy, François Thureau-Dangin et Jean-Vincent Scheil. Né en I827, le premier était un vieux routier de l'assyriologie. Il avait déjà derrière lui une longue liste de publications et une carrière bien établie à l'EPHE. Mais à près de 80 ans, son âge était un obstacle à son recrutement. Professeur de «Langues et Littérature hébraïques, chaldaïques et syriaques », Philippe Berger remarque à l'annonce de la candidature d’Halévy qu'il avait été dans l'ombre de l'ancien titulaire de la chaire, mais que dorénavant il devrait être considéré comme un «initiateur». Si Halévy pouvait paraître trop âgé, Thureau-Dangin, au contraire, était trop jeune. Âgé de 33 ans en 1905, ce conservateur au Louvre commençait à peine sa carrière d’assyriologue. Pour le troisième candidat, la situation était tout autre. Né en I858, le père Scheil appartenait aux intellectuels de l'ordre dominicain. Il avait suivi des études de théologie avant d'entrer en tant qu'élève à l'EPHE en I887. Il eut alors l'occasion de participer à des fouilles en Iran et Irak. Depuis I895, il donnait des cours à l'EPHE, où il devint directeur d'études adjoint en 1902. Ces circonstances plaçaient Fossey et Scheil en position de favoris.

Le procès-verbal de l'assemblée du i7 décembre 1905 permet de constater une mobilisation intense autour de Fossey et de Scheil. Parmi les partisans de Scheil, on trouve Charles Barbier de Meynard (Arabe), Gaston Maspero (Philologie et Archéologie égyptiennes) et Jacques Flach (Histoire des législations comparées), qui soulignent l'expérience du candidat dans ses missions en Orient, ses postes et ses travaux. Dans le camp adverse, on peut compter Berger (Langues et Littérature hébraïques), Réville (Histoire des religions), Havet (Philologie latine), 
Édouard Chavannes (Langue et Littérature chinoise), Chuquet (Langue et Littérature d’origine germanique) et Lévi (Langue et Littérature sanscrites). Ce groupe défendit Fossey avec différents arguments. Réville, par exemple, met en évidence la «culture générale» et le «grand talent de parole» de Fossey; Chavannes, pour sa part, affirme qu'il est le seul à avoir reçu le titre de docteur; enfin, Havet souligne la formation classique de Fossey. Cette avalanche d'arguments s'opposait aux préférences de l’assemblée, acquise à Scheil. En «première ligne», le dominicain fut élu avec 2I voix contre I2, Fossey obtenant l'indication pour la «deuxième ligne» (avec 23 voix contre i3 pour Thureau-Dangin et 2 votes nuls).

C’est pourtant Fossey qui est nommé: une campagne efficace avait été déployée contre la nomination d'un religieux, juste au moment où l'on s'attelait à la séparation de l'Église et de l'État. Le ministre de l'Instruction publique, Jean-Baptiste Bienvenu Martin, inversa l'ordre proposé par l'assemblée, provoquant des réactions dans la presse et chez certains professeurs. Quelques-uns parleront ici de complot socialiste et anticlérical (Besnard, 1979). Le conflit s'étendra au sein du milieu académique par des batailles d'accusations mutuelles entre Fossey et Scheil: dénonciation de plagiat, critiques des travaux du camp adverse. L'AIBL s'engagea elle aussi dans la dispute en prenant le parti de Scheil' ${ }^{13}$.

Le bilan de cette élection au CF est donc en demi-teintes. Parce qu'il incarnait la laïcité, Fossey s'imposa comme le candidat de ceux qui défendaient la fin de l'enseignement confessionnel. Son parcours classique et scientifique put aussi bien plaire aux intellectuels appartenant aux minorités religieuses qu'aux «modernistes $\gg^{14}$ et partisans d'une réforme de l'enseignement. Or, les chercheurs proches de Durkheim se retrouvant dans ce champ, ces affinités ont pu favoriser la cristallisation d'un bloc de professeurs contre les avancées de la sociologie, aux yeux desquels elle apparaissait comme illégitime.

13 L'événement est connu sous le nom de la «Querelle des Orientalistes». Plusieurs documents sont conservée au CF. Voir, à ce propos le dossier C-XIII Fossey.

14 Voir, au sujet des « modernistes », Cholvy et Hilaire, 2000, p. 147-183. 


\subsection{LE DOUBLE ÉCHEC DE MAUSS}

Bien avant d'être élu en I93 I à la chaire de «Sociologie», Mauss s'était déjà présenté deux fois au CF, en 1907 et en 1909 (Fournier, 1994, p. 319-358). C'est la mort d'Albert Réville, titulaire de la chaire d'«Histoire des religions », à la fin de l’année 1906, qui lui offre la première opportunité. Âgé de 35 ans, Mauss était encore jeune, mais il avait déjà acquis une certaine renommée, tant pour ses études sur le sacrifice et la magie que pour ses nombreux comptes rendus sur les thèmes les plus variés de la sociologie religieuse. Après ses études à Bordeaux, sous la supervision de son oncle, il prépare l'agrégation de philosophie, où il est reçu $3^{\mathrm{e}}$ en I895. Suivront deux années d'études à l'EPHE et un stage à l'étranger (Leyde et Oxford). Dès la fin de I90 I, Mauss occupe la chaire de «Religions des peuples non-civilisés» à l'EPHE. Il joue par ailleurs un rôle important au sein du mouvement socialiste. En marque de respect pour Jean Réville, son supérieur à l'EPHE, Mauss, présenté par Meillet et Lévi, demanda à n'être pris en considération que pour la «deuxième ligne».

Les autres candidats à cette chaire vacante étaient Georges Foucart, Maurice Vernes et Jean Réville ${ }^{15}$. Foucart, né en I865, était un spécialiste de la religion de l'Égypte ancienne. Sans être normalien, il avait suivi des études à l'Institut français d’archéologie orientale et enseigné successivement aux Facultés de lettres de Bordeaux et d'Aix-en-Provence. C'est son père lui-même, l'helléniste Paul Foucart, qui était chargé de présenter sa candidature. Vernes avait peu de chances. Ce disciple de Renan, maître de conférences à l'EPHE et cofondateur de la Revue d'histoire des religions, était âgé de 72 ans. Ce fut Louis Havet qui défendit sa candidature. Jean Réville, né en I854, était le fils de l’ancien titulaire. Ancien dreyfusard et pasteur comme son père, ce directeur d'études à l'EPHE, également co-directeur de la Revue d'histoire des religions paraissait le favori de l'élection.

Le procès-verbal de l'assemblée révèle un mouvement en faveur de Mauss mené par Lévi, Meillet, Janet et l'historien Camille Jullian. Mauss est ainsi propulsé comme candidat en «première ligne», sans toutefois disposer du soutien nécessaire pour s'imposer. Au premier tour de l'élection, Réville 
devance ses concurrents avec I4 voix, suivi de Foucart avec Io voix, contre 7 pour Mauss et 5 pour Vernes. Au deuxième tour, les voix se recomposent : Réville obtient 24 voix contre 8 pour Foucart (les autres ne réunissant que 4 voix). Pour la «deuxième ligne», on opposait Mauss à Foucart. En deux tours, Mauss recueille l’appui de la majorité des professeurs (2 I voix contre Io). Fallait-il y voir un signe de reconnaissance de la sociologie?

Un an plus tard, lorsque Jean Réville décède, une seconde opportunité s'ouvrait à Mauss. Il venait d'être nommé directeur d'études à l'EPHE et avait publié son premier livre, Mélanges d'histoire des religions, en collaboration avec Hubert. Mais les candidats se multiplient. Outre ceux de l'élection précédente (Foucart et Vernes), cinq autres se présentent, dont trois visant la «première ligne» : Jules Toutain, Émile Amélineau et Alfred Loisy.

Amélineau et Toutain provenaient de l'EPHE. Âgé de 59 ans, le premier était un spécialiste de la mythologie égyptienne et un archéologue de terrain. Toutain était mieux inséré dans le milieu académique. Normalien et ancien membre de l'École française de Rome, il était devenu un spécialiste des religions anciennes et possédait une solide expérience dans les fouilles de la région méditerranéenne ainsi qu’en épigraphie latine. Il était aussi le traducteur du célèbre Rameau d'or de James Frazer (1890-1915). Loisy, enfin, apparaissait comme une victime de l'Église catholique. Docteur en théologie et directeur de la Revue d'histoire et de littérature religieuses, il était issu d'une mouvance catholique qui fut persécutée après la montée des tensions entre l'Église et l'État. En 1907, il venait d'être excommunié en tant que «moderniste».

Dans ce contexte, Mauss croit à ses chances. À sa famille, il décrit sa campagne en ces termes :

J’ai à peu près fini mes visites. En somme, j’ai II voix sûres [...]. C'est un minimum; le maximum probable c'est I5; peut-être pourra-t-on encore rallier les indifférents, si on en ralliait ce pourrait être le succès. Loisy, lui, en a ıo sûres, mais je ne crois pas qu'il puisse rallier beaucoup de probables [...]. Toutain et Foucart ont 9 voix sûres. Ce sont les candidats réactionnaires. Heureusement qu'ils sont deux. Sans cela, si Toutain était seul, il aurait des chances de passer, bien qu'il soit un imbécile (voir Fournier, 1994, p. 328). 
En effet, il semblait plausible que, dans le cas d'une élection partagée entre Loisy et Mauss, des voix «réactionnaires» se reportent sur le sociologue socialiste juif plutôt que sur le prêtre excommunié. Mais Mauss sousestimait ses handicaps. Par deux lettres de son oncle adressées à Salomon Reinach, on sait qu'il était la cible d'une campagne diffamatoire: il aurait eu des relations avec les partisans de Gustave Hervé, à la tête d'un courant socialiste radical. Durkheim demandait à Reinach de pousser Joseph Bédier et Gabriel Monod, probablement à l'origine des rumeurs, à démentir cette accusation. Il soulignait combien il serait «extraordinaire que la qualité de moine (Scheil) ou de pasteur protestant ne fût pas une objection, et que, seule, celle de socialiste disqualifiât son homme» (Durkheim à Reinach, [1907-1908], voir Benthien, 2010, p. 30). Tel fut pourtant le cas.

Le procès-verbal de l'assemblée du 9 février 1909 permet de retracer les soutiens apportés aux candidats et de dévoiler les alliances élaborées à cette occasion $^{16}$. Foucart fut à nouveau présenté par son père et par Ernest Babelon, autre professeur catholique reconnu pour ses opinions conservatrices. La candidature de Toutain fut appuyée par deux antiquisants, Cagnat et Croiset. Vernes et Amélineau sont introduits par le seul Berger. Enfin, Meillet et Lévi défendent à nouveau Mauss.

Le vote se tient dans un contexte particulièrement tendu. Sur 36 votants, le premier tour donne I2 voix à Foucart, 9 à Loisy, 7 à Mauss, 5 à Toutain, 2 à Vernes et une seule voix à Amélineau. Au second tour, Foucart passe à I 3 voix, Loisy à I I, Mauss à 8 et Toutain à 4 . Toutain écarté, Loisy passe alors en tête avec I7 voix, suivi de Foucart avec I5 voix, Mauss ne conservant que 4 voix. Au quatrième tour, Loisy conserve I7 voix, Foucart passe à 16 voix, 2 voix restant à Mauss, avec un vote nul ${ }^{17}$. Les deux derniers soutiens de Mauss préfèrent alors opter pour l'«excommunié ». Au cinquième et dernier tour, Loisy obtient 19 voix contre 16 pour Foucart.

16 Voir Archives du CF, l'Assemblée du 9 février 1909 (G-IV-g45H), ainsi que le Dossier d'Alfred Loisy (C-XII Loisy).

17 Selon Loisy, le vote nul était celui de Wyrouboff (Loisy, 1913, p. 69). 
Pour l'élection en «deuxième ligne», Paul Foucart, indigné, demande le retrait de la candidature de son fils. Il quitte alors la salle, accompagné de deux professeurs. L'ordre rétabli, le vote reprend son cours et semble d'abord favorable à Mauss, qui obtient I4 voix, contre ıo pour Toutain, 3 pour Vernes et 7 voix réparties entre les autres candidats. Au second tour, les deux professeurs sortis avec Paul Foucart refont leur apparition. Mauss et Toutain se retrouvent à égalité avec I7 voix chacun, Vernes recueillant I voix. Deux personnes quittent de nouveau la salle et l'élection sachève par une courte victoire de Toutain, avec I7 voix contre I6.

Même en tenant compte de la division des «réactionnaires » entre Foucart et Toutain, Mauss n’avait pas réuni l'appui qu'il imaginait, l'«excommunié » gagnant du terrain. Au deuxième tour, comme Mauss l'avait anticipé, le retrait de Foucart avait renforcé Toutain. Son nom suscitait moins de résistance que celui du sociologue juif et socialiste. Quelques mois plus tard, la candidature de Simiand allait à nouveau illustrer le rôle joué par ces variables.

\subsection{SIMIAND: NON AU SOCIALISME, NON À LA SOCIOLOGIE}

Les décès de trois de ses membres, provoquent, au début de 1912, d’intenses débats au CF. Des professeurs sont chargés de rédiger des rapports sur les savoirs qui jusqu'alors n'avaient pas trouvé place dans l'institution et de proposer des noms ${ }^{18}$. Les suggestions sont variées. Entre autres variations sur les mêmes thèmes, citons : «Ethnographie» (Arnold Van Gennep), «Faits Économiques et Sociaux » (pas de nom consensuel), «Géographie humaine» (Jean Bruhnes), «Histoire de l'Afrique du Nord» (Stéphane Gsell), «Histoire de la Marine et de l'Expansion coloniale de la France» (Charles de la Roncière), «Statistique économique» (pas de nom consensuel), enfin «Théorie et Histoire de l'histoire» (Henri Berr). Pour décider des choix à faire, une série de votes fut organisée le 2 I janvier. Dès qu’une proposition réunissait une majorité absolue, on passait à un autre vote pour les choix restants. Trois créations de chaires sont ainsi retenues: «Étude des faits économiques et sociaux», «Histoire de l’Afrique du Nord» et «Géographie Humaine». 
Pour le projet «Faits économiques et sociaux», qui nous intéresse ici, on remarque d'abord l'absence de noms consensuels. Les professeurs chargés d’en rédiger le rapport sont Leroy-Beaulieu, Izoulet et Georges Renard, un professeur (Histoire du travail) imposé par l'État en 1907. Leur difficulté à atteindre un consensus n'est pas accidentelle puisque les deux premiers, connus comme des penseurs libéraux, devaient faire bon ménage avec un socialiste. D’ailleurs, si ce projet est retenu, c'est sans doute que tous pouvaient aspirer à y présenter leur propre candidat.

Toujours ce 2i janvier, Renard évoque déjà les compétences de Simiand, à ses yeux le candidat le plus qualifié. Les seuls à se prononcer alors sont Bergson et Jullian. Le premier affiche d'emblée son soutien à Simiand, qu'il décrit comme «un esprit des plus vigoureux, des plus pénétrants et des plus philosophiques »; le second estime que Marcel Marion est le candidat le plus approprié.

Deux mois plus tard, le 24 mars, l'élection se déroule au cours d'une séance tumultueuse ${ }^{19}$. L'absence de favoris avait multiplié les espoirs et huit candidats se présentent en «première ligne». Parmi eux, Marion, Louis Germain-Martin et Simiand font l'objet d'expressions de sympathie à l’assemblée, dénotant des campagnes bien menées.

Jullian défend à nouveau la candidature de Marion, son camarade à l'ENS et ancien collègue à l'université de Bordeaux. Catholique et conservateur sur les questions économiques, Marion s'est distingué par la suite comme l'un des principaux partisans du libéralisme orthodoxe en France. Leroy-Beaulieu donne son appui à Louis Germain-Martin. Cet ancien chartiste et agrégé de droit, professeur en province, se consacre alors à l'étude du développement industriel, notamment sous l'Ancien Régime. Renard, enfin, présente de nouveau Simiand, normalien, sociologue et socialiste. Il met en avant l'importance de ses travaux et son rôle de «représentant de la jeune école» durkheimienne. Simiand occupait alors un poste à l'EPHE, après avoir été pendant des années chargé de mission auprès du Ministère du commerce et du travail. Lévi, évoquant déjà le nom d’Henri Bourgin pour la «deuxième ligne», déclare son soutien à Simiand. 
Un incident de procédure traduit cependant l'aversion de certains membres de l'assemblée pour les «sociologues». Dans sa défense de Simiand, Renard avait pris la liberté de critiquer les travaux d'un autre candidat. Les réactions déclenchées font bien ressortir les éléments extra-intellectuels qui structurent la perception sociale des candidats :

M. Renard ajoute que, ayant été rapporteur des titres de plusieurs candidats et par là chargé de confronter leurs mérites, il croit devoir dire pourquoi il ne peut, même en seconde ligne, voter pour M. Germain Martin. Il croit que ce candidat n’a que des qualités moyennes et manque surtout d'originalité, et il sattache à montrer, par quelques exemples, que M. Germain Martin écrit souvent d'une façon défectueuse.

M. Leroy-Beaulieu regrette que les ouvrages de M. Germain Martin soient censurés d'un point de vue si spécial; [...] il voudrait qu’on en revînt à l'ancien usage d'après lequel on s'abstenait le plus possible de critiquer directement les candidats patronnés par d'autres professeurs.

M. Foucart reproche à M. Simiand un abus des préoccupations méthodologiques et philosophiques; il reconnaît, dit-il, dans ses travaux, les travers et la phraséologie de l'école sociologique. Il indique en outre que M. Simiand a enseigné [...] à l'École socialiste.

M. Renard proteste contre cette dernière observation qui tendrait à faire intervenir des considérations politiques dans une question purement scientifique. Il regrette que M. Foucart fasse grief à un candidat d'avoir enseigné à l'École socialiste, et qu'il lui fasse ce grief en présence d'un collègue qui lui-même a pris ouvertement la qualité de socialiste et s'en fait honneur [...].

M. Bergson [...] insiste sur le tour philosophique de son esprit; cette aptitude philosophique n'est pas [...] un défaut, mais bien une qualité, indispensable dans l'observation et dans la critique des faits sociaux (Voir Archives du CF, l'Assemblée du 24 mars 1912 [G-IV-g II]).

Les votes consomment alors une double défaite pour Simiand. En «première ligne», il est devancé en trois tours par Marion (23 voix contre I3). Quant à la «deuxième ligne», c'est Germain-Martin qui l'emporte (I8 voix contre I7). À cette dernière occasion qui se présentait avant la guerre, les sociologues ne réussirent pas à rassembler beaucoup plus que leurs habituels fidèles. En aurait-il été autrement sans la maladresse de Renard ? L'événement aura en tout cas montré le poids des arguments d'ordre politique, mis sur le même plan que celui du savoir. 


\section{CONCLUSION Science et Politique}

Dans un contexte d'ouverture à des savoirs nouveaux, qui pouvait leur paraître favorable, les durkheimiens se sont donc heurtés à une hostilité certaine: sur sept tentatives, seule une seule victoire fut incontestée (Meillet), la seconde prêtant le flanc à la critique (Fossey). L'analyse des registres argumentaires déployés fait d’ailleurs ressortir des régularités. Sur le plan intellectuel, on s'appuie sur l'institutionnalisation précaire de la sociologie et sur le caractère polémique de ses objets d'étude pour avancer que cette nouvelle science paraît «peu rigoureuse». Il ne restait donc aux durkheimiens que la possibilité de s’infiltrer au CF par le biais de spécialités plus légitimes.

Les réussites du groupe furent d’ailleurs facilitées par leur investissement dans des disciplines déjà reconnues. C'est en dépit de leur implication dans la sociologie que Meillet et Fossey sont élus, grâce à la notoriété qu'ils ont acquise respectivement en tant que linguiste et assyriologue. En revanche, Durkheim, Mauss et Simiand empruntaient des voies plus compliquées en postulant pour des chaires également convoitées par des philosophes, des théologiens, des économistes ou des historiens. Ce n'est pas un hasard si Mauss doit l'élan initial de sa carrière à une spécialisation (celle des «religions primitives »); Simiand, à une carrière en grande partie extra-universitaire; Durkheim, à une intervention de nature politique (sa nomination à Bordeaux). On voit donc ici que l'autonomie des sociologues par rapport à la position institutionnelle précaire de la sociologie fut, paradoxalement, un élément fondamental de leurs succès.

Cela dit, on peut constater la formation, parmi les professeurs, d'un groupe de sympathisants de la sociologie. Ce cercle regroupait surtout des spécialistes en diverses civilisations, tels que l'indianiste Lévi ou Fossey lui-même. Ils voyaient dans la sociologie des partenaires possibles pour faire avancer leurs propres champs d'études. Au sein de l'ASMP, les sociologues recevaient par ailleurs le soutien de représentants de disciplines se montrant ouvertes aux procédures scientifiques. C’est le cas de Bergson ou de Janet. Avec le temps, leurs rangs s'étofferont au CF avec Renard en 1907 et Édouard Fuster en 1917, nommés sur décision politique, ou avec le géographe Jean Brunhes, élu en I9ı2. Enfin, même si 
les scientifiques ne se prononçaient que rarement aux élections des chaires de lettres, quelques-uns apporteront aux durkheimiens leur soutien. On mentionnera ici Wyrouboff, leur seul allié explicite, ainsi que le biologiste Émile Gley, qui entretint des relations avec Durkheim (Fournier, 1994, p. 327).

Mais pour la majorité des autres groupes de professeurs, les liens avec les sociologues ont été beaucoup plus ambivalents. Pour les historiens et spécialistes en langues et littératures, notamment les représentants des disciplines centrales - surtout le français et le latin -, la situation dépendait de la non-mise en question de leur position hégémonique. La fin du monopole de la formation classique dans l'enseignement secondaire en 1902 semble avoir poussé ces universitaires à une posture défensive. Entre 1897 et 1918, trois nouvelles chaires viennent recouper en grande partie les chaires déjà existantes : «Histoire et Antiquités nationales» (1905), «Numismatique de l'Antiquité et du Moyen Âge» (1908) et «Histoire de l’Afrique du Nord» (I9I2). On observe alors que les «littéraires » classiques ne contrent les projets favorables à la sociologie que lorsqu'ils menaçaient les leurs. Quant au groupe d'enseignants représentant l'ASMP, ils auront manifesté une résistance continue, qu'il s’agisse de défenseurs théoriques de l'individualisme et du libéralisme (Izoulet et Tarde) ou de philosophes de formation chrétienne (Loisy).

Au-delà des arguments intellectuels, ces analyses font enfin ressortir l'articulation entre ces recrutements et la politique. En effet, si la sociologie durkheimienne disposait d'une relative autonomie, elle apparaissait toujours, aux yeux de ses contemporains, liée à un projet politique, laïque pour les uns, socialiste pour les autres. Ce lien aura compromis la légitimité des sociologues. La première tentative de Durkheim fut à cet égard paradigmatique : la nomination politique d'Izoulet était justifiée par la chasse aux socialistes et les calomnies lancées contre Fossey, Mauss ou Simiand illustrent le même phénomène.

Les tentatives postérieures à 1918 feront apparaître les mêmes régularités. L'élection, en 1923, d'Alexandre Moret, égyptologue qui participa à la deuxième série de L’Année sociologique, fut symptomatique puisqu'il s'agissait encore une fois d'un spécialiste. Mauss et Simiand devront attendre la réorganisation des gauches pour, dans des élections là aussi très serrées, voir leurs candidatures aboutir. Le cas d’Isidore Lévy, élu en 1933 pour la chaire d’ «Histoire ancienne 
de l'orient sémitique », aura été plus facile. Dans le contexte dramatique de la Seconde Guerre mondiale, André Piganiol, historien de Rome, et Halbwachs seront les derniers durkheimiens élus au CF. Après 1945, ni la politique, ni l'université, ni les sciences sociales ne seront les mêmes.

\section{BIBLIOGRAPHIE*}

BENTHIEN Rafael Faraco, 2010, «Lettres d'Émile Durkheim à Salomon Reinach», Durkheimian Studies, 16, p. 19-35.

BESNARD Philippe, 1979, «Durkheim, les durkheimiens et le Collège de France», Études Durkheimiennes, Ire s., 3, p. 4-7.

BOURCIEZ Édouard, 1906, «Un manifeste de la nouvelle école linguistique», Revue des études anciennes, 8, p. 272-274.

BROOKS John I., 2002, «Institutionalizing Durkheimian Sociology of Religion: The Case of the Fifth Section», Thinking about religion, 2 [en ligne]: $<$ http://organizations.uncfsu.edu/ncrsa/journal/v02/johnbrooks.htm>.

CHANTEPIE DE LA SAUSSAYE Pierre Daniel, 1904, Manuel d'Histoire des Religions, Paris, Armand Colin.

CHARLE Christophe, 1985, Dictionnaire biographique des universitaires aux XIX et $x x^{e}$ siècles. Vol. I. La Faculté des Lettres de Paris (1809-1908), Paris, Éd. du CNRS.

-, 1997, «Le Collège de France», in Les Lieux de mémoire, t. 3, Les France, Pierre Nora (dir.), Paris, Gallimard, p. 1983-2008.

- et TELKÈS Eva, 1988, Les Professeurs du Collège de France, Paris, Éd. du CNRS/ INRP.

CHOLVY Gérad et HILAIRE Yves-Marie (dir.), 2000, Histoire religieuse de la France, 1880-19/4, Toulouse, Privat.

CONSOLIM Marcia Cristina, 2008, «Gabriel Tarde e as ciências sociais francesas: afinidades eletivas», Mana (Rio de Janeiro), 14-2, p. 269-298.

* Nous n'avons pas inséré dans la bibliographie les références inédites concernant les archives du Collège de France. Les cotes des documents sont donc indiquées en notes de bas de page. 
DURKHEIM Émile, 1998, Lettres à Marcel Mauss, Paris, PUF.

-, 1999 (1904-1905), L'Évolution pédagogique en France, Paris, PUF.

FOURNIER Marcel, 1994, Marcel Mauss, Paris, Fayard.

-, 2007, Émile Durkheim, 1858-1917, Paris, Fayard.

GRELL Chantal, 1995, Le Dix-huitième Siècle et l'antiquité en France, 1680-1789, 2 tomes, Oxford, Voltaire Fondation.

HALBWACHS Maurice, 1999, «Ma campagne au Collège de France», Revue d'Histoire des Sciences Humaines, I-I, p. I89-229.

HEILBRON Johan, 2006, La Naissance de la sociologie en France, Paris, Agone.

KARADY Victor, 1976, «Durkheim, les sciences sociales et l'Université», Revue française de sociologie, 17, p. 267-311.

-, 1979, «Stratégies de réussite et modes de faire-valoir de la sociologie chez les durkheimiens», Revue française de sociologie, 20, p. 49-82.

LEFRANC Abel, 1893, Histoire du Collège de France: depuis ses origines jusqu'à la fin du premier Empire, Paris, Hachette.

LOISY Alfred, 1913, Choses passées, Paris, Nourry.

LUKES Steven, 1973, Émile Durkheim, His Life and Work: a Historical and Critical Study, Londres, Lane.

MEILLET Antoine, 1948, Linguistique historique et linguistique générale, Paris, Honoré Champion.

MUCCHIELLI Laurent, 1998, La Découverte du social: naissance de la sociologie en France (1870-1914), Paris, La Découverte.

RINGER Fritz K., 1992, Fields of Knowledge: French Academic Culture in Comparative Perspective, 1890-1920, Cambridge, Cambridge University Press.

SABATIER Auguste, 1898, «Lettre du Dimanche. Un nouveau cours au Collège de France - M. J. Izoulet et la philosophie sociale - Les quatre problèmes du temps présent», Revue chrétienne, 45-I, p. 373-377.

STRENSKI Ivan, 2000, «Durkheimians and Protestants in the École Pratique, 5e Section: The Dark Side», Durkheimian Studies, 6, p. I05-II4. 